\title{
Two peculiar fast transients in a strongly lensed host galaxy
}

\author{
S. A. Rodney $\oplus^{1 \star}$, I. Balestra ${ }^{2}$, M. Bradac ${ }^{3}$, G. Brammer ${ }^{4}$, T. Broadhurst ${ }^{5,6}$, G. B. Caminha $\oplus^{7,8}$, G. Chirivi ${ }^{9}$, \\ J. M. Diego ${ }^{10}$, A. V. Filippenko ${ }^{11,12}$, R. J. Foley ${ }^{13}$, O. Graur ${ }^{14,15,16}$, C. Grillo ${ }^{17,18}$, S. Hemmati ${ }^{19}$, J. Hjorth ${ }^{18}$, \\ A. Hoag ${ }^{3}{ }^{3}$, M. Jauzac ${ }^{20,21,22}$, S. W. Jha ${ }^{23}$, R. Kawamata ${ }^{24}$, P. L. Kelly ${ }^{11,25}$, C. McCully 26,27 , B. Mobasher ${ }^{28}$, \\ A. Molino ${ }^{29,30}$, M. Oguri ${ }^{31,32,33}$, J. Richard ${ }^{34}$, A. G. Riess ${ }^{4,35}$, P. Rosati ${ }^{7}$, K. B. Schmidtt ${ }^{27,36}$, J. Selsing ${ }^{18}$, \\ K. Sharon ${ }^{37}$, L.-G. Strolger ${ }^{4}$, S. H. Suyu ${ }^{9,38,39}$, T. Treu ${ }^{40}$, B. J. Weiner ${ }^{41}$, L. L. R. Williams ${ }^{25}$ and A. Zitrin ${ }^{42}$
}

\footnotetext{
'Department of Physics and Astronomy, University of South Carolina, Columbia, SC, USA. ${ }^{2}$ University Observatory Munich, Munich, Germany. ${ }^{3}$ Department of Physics, University of California, Davis, CA, USA. ${ }^{4}$ Space Telescope Science Institute, Baltimore, MD, USA. ${ }^{5}$ Fisika Teorikoa, Zientzia eta Teknologia Fakultatea, Euskal Herriko Unibertsitatea UPV/EHU, Leioa, Spain. ${ }^{6}$ IKERBASQUE, Basque Foundation for Science, Alameda Urquijo, Bilbao, Spain. ${ }^{7}$ Dipartimento di Fisica e Scienze della Terra, Università degli Studi di Ferrara, Ferrara, Italy. ${ }^{8}$ Kapteyn Astronomical Institute, University of Groningen, P.O. Box 800, 9700 AV Groningen, The Netherlands. ${ }^{9}$ Max-Planck-Institut für Astrophysik, Garching, Germany. ${ }^{10}$ IFCA, Instituto de Física de Cantabria (UC-CSIC), Santander, Spain. "'Department of Astronomy, University of California, Berkeley, CA, USA. ${ }^{12}$ Miller Institute for Basic Research in Science, University of California, Berkeley, CA, USA. ${ }^{13}$ Department of Astronomy and Astrophysics, University of California, Santa Cruz, CA, USA. ${ }^{14}$ Center for Cosmology and Particle Physics, New York University, New York, NY, USA. ${ }^{15}$ Department of Astrophysics, American Museum of Natural History, New York, NY, USA. ${ }^{16} \mathrm{Harvard} /$ Smithsonian Center for Astrophysics, Cambridge, MA, USA. ${ }^{17}$ Dipartimento di Fisica, Università degli Studi di Milano, Milan, Italy. ${ }^{18}$ Dark Cosmology Centre, Niels Bohr Institute, University of Copenhagen, Copenhagen, Denmark. ${ }^{19}$ Infrared Processing and Analysis Center, California Institute of Technology, Pasadena, CA, USA. ${ }^{\circ}$ Centre for Extragalactic Astronomy, Department of Physics, Durham University, Durham, UK. ${ }^{21}$ Institute for Computational Cosmology, Durham University, Durham, UK. ${ }^{22}$ Astrophysics and Cosmology Research Unit, School of Mathematical Sciences, University of KwaZulu-Natal, Durban, South Africa. ${ }^{23}$ Department of Physics and Astronomy, Rutgers, The State University of New Jersey, Piscataway, NJ, USA. ${ }^{24}$ Department of Astronomy, Graduate School of Science, The University of Tokyo, Tokyo, Japan. ${ }^{25}$ School of Physics and Astronomy, University of Minnesota, Minneapolis, MN, USA. ${ }^{26}$ Las Cumbres Observatory Global Telescope Network, Goleta, CA, USA. ${ }^{27}$ Department of Physics, University of California, Santa Barbara, CA, USA. ${ }^{28}$ Department of Physics and Astronomy, University of California, Riverside, CA, USA. ${ }^{29}$ Universidade de São Paulo, Cidade Universitária, Instituto de Astronomia, Geofísica e Ciências Atmosféricas, São Paulo, Brazil. ${ }^{30}$ Instituto de Astrofísica de Andalucía (CSIC), Granada, Spain. ${ }^{31}$ Research Center for the Early Universe, University of Tokyo, Tokyo, Japan. ${ }^{32}$ Department of Physics, University of Tokyo, Tokyo, Japan. ${ }^{33} \mathrm{Kavli}$ Institute for the Physics and Mathematics of the Universe (Kavli IPMU, WPI), University of Tokyo, Kashiwa, Japan. ${ }^{34}$ Univ Lyon, Univ Lyon1, Ens de Lyon, CNRS, Centre de Recherche Astrophysique de Lyon UMR5574, F-69230 Saint-Genis-Laval, France. ${ }^{35}$ Department of Physics and Astronomy, The Johns Hopkins University, Baltimore, MD, USA. ${ }^{36}$ Leibniz-Institut für Astrophysik Potsdam (AIP), Potsdam, Germany. ${ }^{37}$ Department of Astronomy, University of Michigan, Ann Arbor, MI, USA. ${ }^{38}$ Institute of Astronomy and Astrophysics, Academia Sinica, Taipei, Taiwan. ${ }^{39}$ Physik-Department, Technische Universität München, Garching, Germany. ${ }^{40}$ Department of Physics and Astronomy, University of California, Los Angeles, CA, USA. ${ }^{41}$ Department of Astronomy, University of Arizona, Tucson, AZ, USA. ${ }^{42}$ Ben-Gurion University of the Negev, Beer-Sheva, Israel. *e-mail: srodney@sc.edu
} 


\section{Two Peculiar Fast Transients in a Strongly Lensed Host Galaxy}

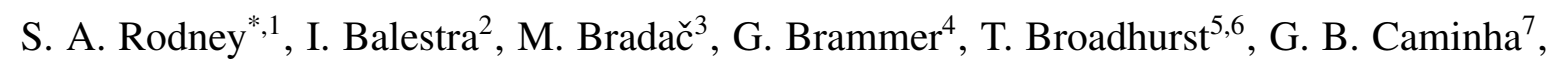

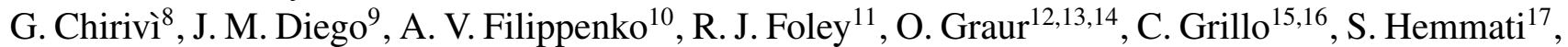
J. Hjorth $\frac{16}{16}$ A. Hoag ${ }^{3}$, M. Jauzad $\frac{18 \mid 1920}{2}$, S. W. Jha ${ }^{21}$, R. Kawamata ${ }^{22}$, P. L. Kelly $\stackrel{10}{10}$ C. McCully $23 / 24$,

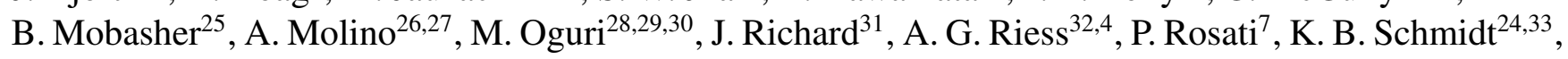

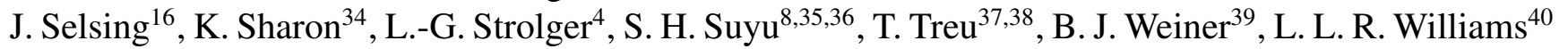
\& A. Zitrin 41

*Corresponding author

${ }^{1}$ Department of Physics and Astronomy, University of South Carolina, 712 Main St., Columbia, SC 29208, USA

${ }^{2}$ University Observatory Munich, Scheinerstrasse 1, D-81679 Munich, Germany

${ }^{3}$ University of California Davis, 1 Shields Avenue, Davis, CA 95616

${ }^{4}$ Space Telescope Science Institute, 3700 San Martin Dr., Baltimore, MD 21218, USA

${ }^{5}$ Fisika Teorikoa, Zientzia eta Teknologia Fakultatea, Euskal Herriko Unibertsitatea UPV/EHU

${ }^{6}$ IKERBASQUE, Basque Foundation for Science, Alameda Urquijo, 36-5 48008 Bilbao, Spain

${ }^{7}$ Dipartimento di Fisica e Scienze della Terra, Università degli Studi di Ferrara, via Saragat 1, I-44122, Ferrara, Italy

${ }^{8}$ Max-Planck-Institut für Astrophysik, Karl-Schwarzschild-Str. 1, 85748 Garching, Germany

${ }^{9}$ IFCA, Instituto de Física de Cantabria (UC-CSIC), Av. de Los Castros s/n, 39005 Santander, Spain

${ }^{10}$ Department of Astronomy, University of California, Berkeley, CA 94720-3411, USA

${ }^{11}$ Department of Astronomy and Astrophysics, University of California, Santa Cruz, CA 95064, USA

${ }^{12}$ Center for Cosmology and Particle Physics, New York University, New York, NY 10003, USA

${ }^{13}$ Department of Astrophysics, American Museum of Natural History, Central Park West and 79th Street, New York, NY 10024, USA

${ }^{14}$ Harvard/Smithsonian Center for Astrophysics, Cambridge, MA 02138, USA

${ }^{15}$ Dipartimento di Fisica, Università degli Studi di Milano, via Celoria 16, I-20133 Milano, Italy

${ }^{16}$ Dark Cosmology Centre, Niels Bohr Institute, University of Copenhagen, Juliane Maries Vej 30, DK-2100 Copenhagen, Denmark

${ }^{17}$ Cahill Center for Astronomy and Astrophysics, California Institute of Technology, MC 249-17, Pasadena, CA 91125, USA

${ }^{18}$ Centre for Extragalactic Astronomy, Department of Physics, Durham University, Durham DH1 3LE, U.K.

${ }^{19}$ Institute for Computational Cosmology, Durham University, South Road, Durham DH1 3LE, U.K.

${ }^{20}$ Astrophysics and Cosmology Research Unit, School of Mathematical Sciences, University of KwaZulu-Natal, Durban 4041, South Africa

${ }^{21}$ Department of Physics and Astronomy, Rutgers, The State University of New Jersey, Piscataway, NJ 08854, USA

${ }^{22}$ Department of Astronomy, Graduate School of Science, The University of Tokyo, 7-3-1 Hongo, Bunkyo-ku, Tokyo 113-0033, Japan

${ }^{23}$ Las Cumbres Observatory Global Telescope Network, 6740 Cortona Dr., Suite 102, Goleta, California 93117, USA

${ }^{24}$ Department of Physics, University of California, Santa Barbara, CA 93106-9530, USA

${ }^{25}$ Department of Physics and Astronomy, University of California, Riverside, CA 92521, USA

${ }^{26}$ Instituto de Astronomia, Geofísica e Ciências Atmosféricas, Universidade de São Paulo, Cidade Universitária, 05508-090, São Paulo, Brazil

${ }^{27}$ Instituto de Astrofísica de Andalucía (CSIC), E-18080 Granada, Spain

${ }^{28}$ Research Center for the Early Universe, University of Tokyo, 7-3-1 Hongo, Bunkyo-ku, Tokyo 113-0033, Japan

${ }^{29}$ Department of Physics, University of Tokyo, 7-3-1 Hongo, Bunkyo-ku, Tokyo 113-0033, Japan

${ }^{30}$ Kavli Institute for the Physics and Mathematics of the Universe (Kavli IPMU, WPI), University of Tokyo, 5-1-5 Kashiwanoha, Kashiwa, Chiba 277-8583, Japan

${ }^{31}$ Université Lyon, Univ Lyon1, Ens de Lyon, CNRS, Centre de Recherche Astrophysique de Lyon UMR5574, F69230, Saint-Genis-Laval, France 
${ }^{32}$ Department of Physics and Astronomy, The Johns Hopkins University, 3400 N. Charles St., Baltimore, MD 21218, USA

${ }^{33}$ Leibniz-Institut für Astrophysik Potsdam (AIP), An der Sternwarte 16, 14482 Potsdam, Germany

${ }^{34}$ Department of Astronomy, University of Michigan, 1085 S. University Avenue, Ann Arbor, MI 48109, USA

${ }^{35}$ Institute of Astronomy and Astrophysics, Academia Sinica, P.O. Box 23-141, Taipei 10617, Taiwan

${ }^{36}$ Physik-Department, Technische Universität München, James-Franck-Straße 1, 85748 Garching, Germany

${ }^{37}$ Department of Physics and Astronomy, University of California, Los Angeles, CA 90095

${ }^{38}$ Packard Fellow

${ }^{39}$ Department of Astronomy, University of Arizona, Tucson, AZ 85721, USA

${ }^{40}$ School of Physics and Astronomy, University of Minnesota, 116 Church Street SE, Minneapolis, MN 55455, USA

${ }^{41}$ Ben-Gurion University of the Negev P.O.B. 653 Beer-Sheva 8410501, Israel 


\section{Supplementary Information}

Lens Model Variations. Supplementary Figure 1 presents probability distributions for the three magnifications and two time delay values of interest. These distributions were derived by combining the Monte Carlo chains from the CATS, GLAFIC, GLEE, and ZLTM models, and individual runs of the GRALE model, which uses a different random seed for each run. We applied a weight to each model to account for the different number of model iterations used by each modeling team. All five of these models agree that host image 11.3 is the leading image, appearing some 3-7 years before the other two images. The models do not agree on the arrival sequence of images 11.1 and 11.2: some have the NW image 11.2 as a leading image, and others have it as a trailing image. However, the models do consistently predict that the separation in time between those two images should be roughly in the range of 1 to 60 days.

Because of the proximity of the critical curves in all models, the predicted time delays and magnification factors are significantly different if calculated at the model-predicted positions instead of the observed positions. For example, in the GLEE model series (GLEE and GLEE-var) when switching from the observed to model-predicted positions the arrival order of the NW and SE images flips, the expected time delay drops from tens of days to $<1$ day, and the magnifications change by $30-60 \%$. However, the expected magnifications and time delays between the events still fall within the broad ranges summarized in Table 1 in the main text and shown in Supplementary Figure 1. Regardless of whether the model predictions are extracted at the observed or predicted positions of the HFF14Spo events, none of the lens models can accommodate the observed 234-day time difference as purely a gravitational lensing time delay.

We used variations of several lens models to investigate how the lensing critical curves shift under a range of alternative assumptions or input constraints. These variations highlight the range of systematic effects that might impact the model predictions for the HFF14Spo magnifications, time delays and proximity to the critical curves. Figure 3 in the main text shows the critical curves for a source at $z=1$ (the redshift of the HFF14Spo host galaxy) predicted by our seven baseline models, plus the four variations described below. Within a given model, variations that move a critical curve closer to the position of HFF14Spo-NW would drive the magnification of that event much higher (toward $\mu_{\mathrm{NW}} \approx 200$ ). This generally also has the effect of moving the critical curve farther from HFF14Spo-SE, which would necessarily drive its magnification downward (toward $\left.\mu_{\mathrm{SE}} \approx 10\right)$.

The baseline CATS model reported in Table 1 in the main text corresponds to the CATSv4.1 model published on the STScI Frontier Fields lens model repository (https://archive. st sci.edu/pub/hlsp/frontier/macs $0416 /$ models/cats/v4.1/). That model uses 178 cluster member galaxies, including a galaxy $<5^{\prime \prime}$ south of the HFF14Spo host galaxy, which creates a local critical curve that intersects the HFF14Spo-SE location. Our CATS-var model is an earlier iteration of the model, published on the STScI repository as CATSv4 (https:// archive.stsci.edu/pub/hlsp/frontier/macs0416/models/cats/v4/), and includes only 98 galaxies identified as cluster members. In this variation the nearby cluster member 


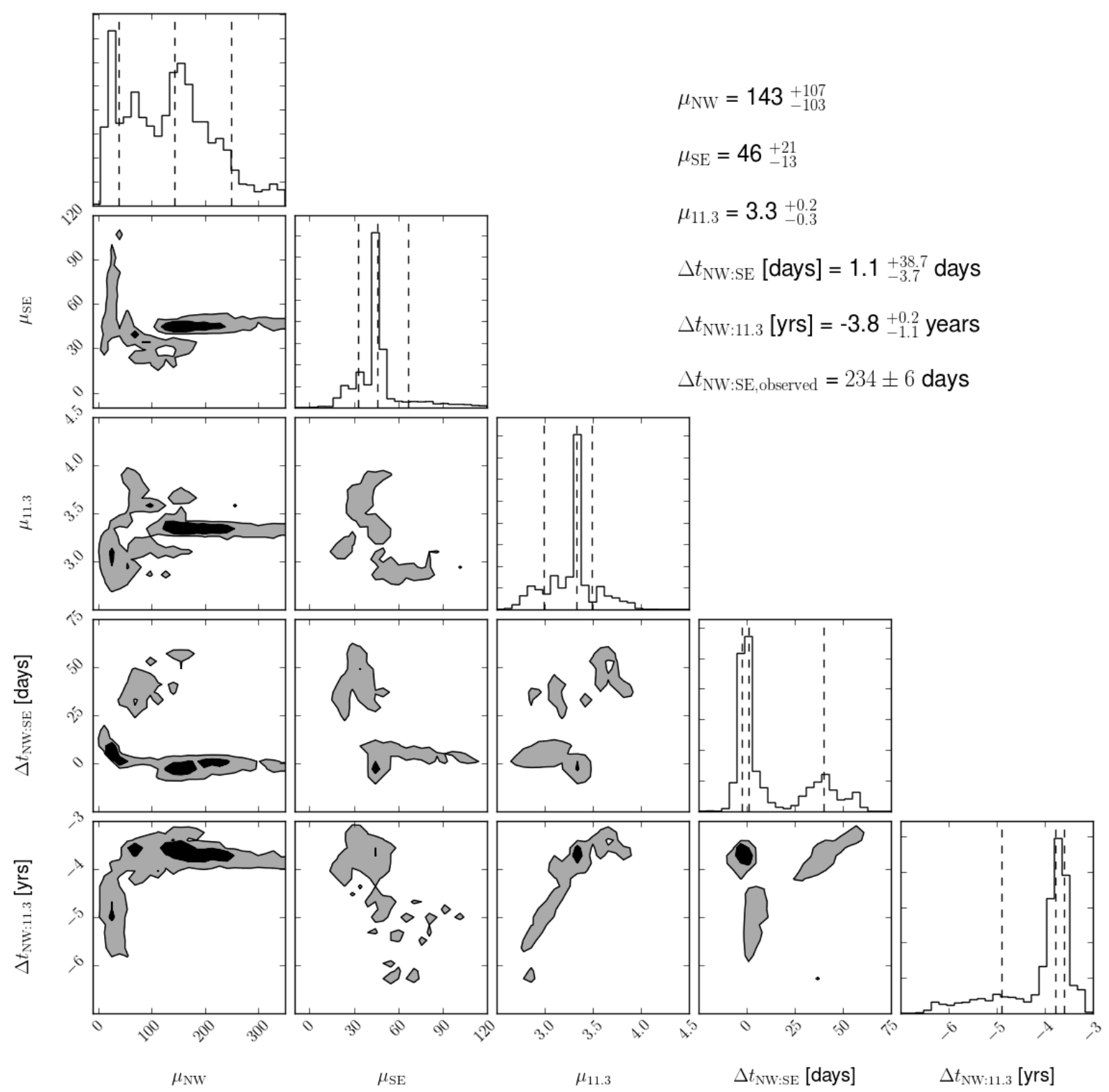

Supplementary Figure 1: Probability distributions for the five primary magnification and timedelay observables, drawn from a combination of results from five of our seven baseline lens models: CATS, GLAFIC, GLEE, GRALE, and ZLTM. Contours shown in the ten panels at the lower left mark the $1 \sigma$ and $2 \sigma$ confidence regions in each two-dimensional slice of the parameter space. Histograms at the top of each column show the marginalized 1-D probability distributions, with dashed vertical lines marking the mean and $1 \sigma$ confidence region. These mean values and uncertainties are also reported in the table of values at the upper right. The final line in the table reports the observed time gap in days between HFF14Spo-NW in January, 2014 and HFF14Spo-SE in August, 2014. 
galaxy is not included, so the HFF14Spo-SE event is not intersected by a critical curve. However, the HFF14Spo-NW event is approximately coincident with the primary critical curve of the MACS0416 cluster. When the critical curve is close to either HFF14Spo location, the magnifications predicted by the CATS model are driven up to $\mu>100$. However, the time delays remain small, on the order of tens of days, and incompatible with the observed 234-day gap.

The WSLAP-var model evaluates whether the cluster redshift significantly impacts the positioning of the critical curve. In this merging cluster, the northern brightest cluster galaxy (BCG) has a slighter higher redshift than the southern BCG. The mean redshift of the cluster is not precisely determined, since it is likely to be aligned somewhat along the line of sight. For the WSLAP-var model we shift the assumed cluster redshift $z=0.4$ from the default $z=0.396$ (used in all the baseline models). The shift in the critical curve is noticeable, but not substantial, insofar as this change does not drive the critical curve to intersect either or both of the HFF14Spo locations.

The GLEE-var model is a multi-plane lens model (Chirivì et al., in prep.) that incorporates 13 galaxies with spectroscopic redshifts that place them either in the foreground or background of the MACS0416 cluster. Supplementary Figure 2 marks these 13 galaxies and highlights two of them that appear in the foreground of the HFF14Spo host galaxy and are close to the lines of sight to the HFF14Spo transients. Both the foreground $z=0.0557$ galaxy and the reconstructed position of the $z=0.9397$ galaxy have a projected separation of $<4$ " from the HFF14Spo-SE transient position. Including these galaxies in the GLEE lensing model changes the absolute value of the magnifications at the location of HFF14Spo-NW (HFF14Spo-SE) to $\sim 70(\sim 250)$ and the time delay between the two locations to $\sim 50$ days. The line-of-sight galaxies also result in a shift of the position of the critical curve - as can be seen by comparing the GLEE and GLEE-var models in Figure 3 in the main text. Nonetheless, the predicted time delays are still incompatible with the observed gap of 234 days between events.

The GLAFIC-var model examines whether it is plausible for a critical curve to intersect both HFF14Spo locations - contrary to the baseline assumption of a single critical curve subtending the HFF14Spo host galaxy roughly midway between the two positions. This model includes a customized constraint, requiring that the magnification factors at the HFF14Spo positons are $>$ 1000. To achieve this, we independently adjusted the mass scaling for the two nearest cluster member galaxies, which are located just northeast and south of the HFF14Spo host galaxy arc. The mass of the northeast member galaxy was increased by $\sim 30 \%$ and that of the southern one by $\sim 60 \%$. As a simple check of the predicted morphology of the host galaxy, we placed a source with a simple Sersic profile ${ }^{2}$ on the source plane. The lensed image of that artificial source is an unbroken elongated arc, reproducing the host galaxy image morphology reasonably well.

For this modification of the GLAFIC lens model to be justified in a statistical sense, the revised model should still accurately reproduce the observed strong-lensing constraints across the entire cluster. The $\chi^{2}$ statistic for the baseline GLAFIC model is 240, with 196 degrees of freedom $\left(\chi_{\nu}^{2}=1.2\right)$, and yields an Akaike information criterion (AIC) $)^{3}$ of 676. For the GLAFIC-var model that forces multiple critical curves to intersect the HFF14Spo locations, we get $\chi^{2}=331$ for 


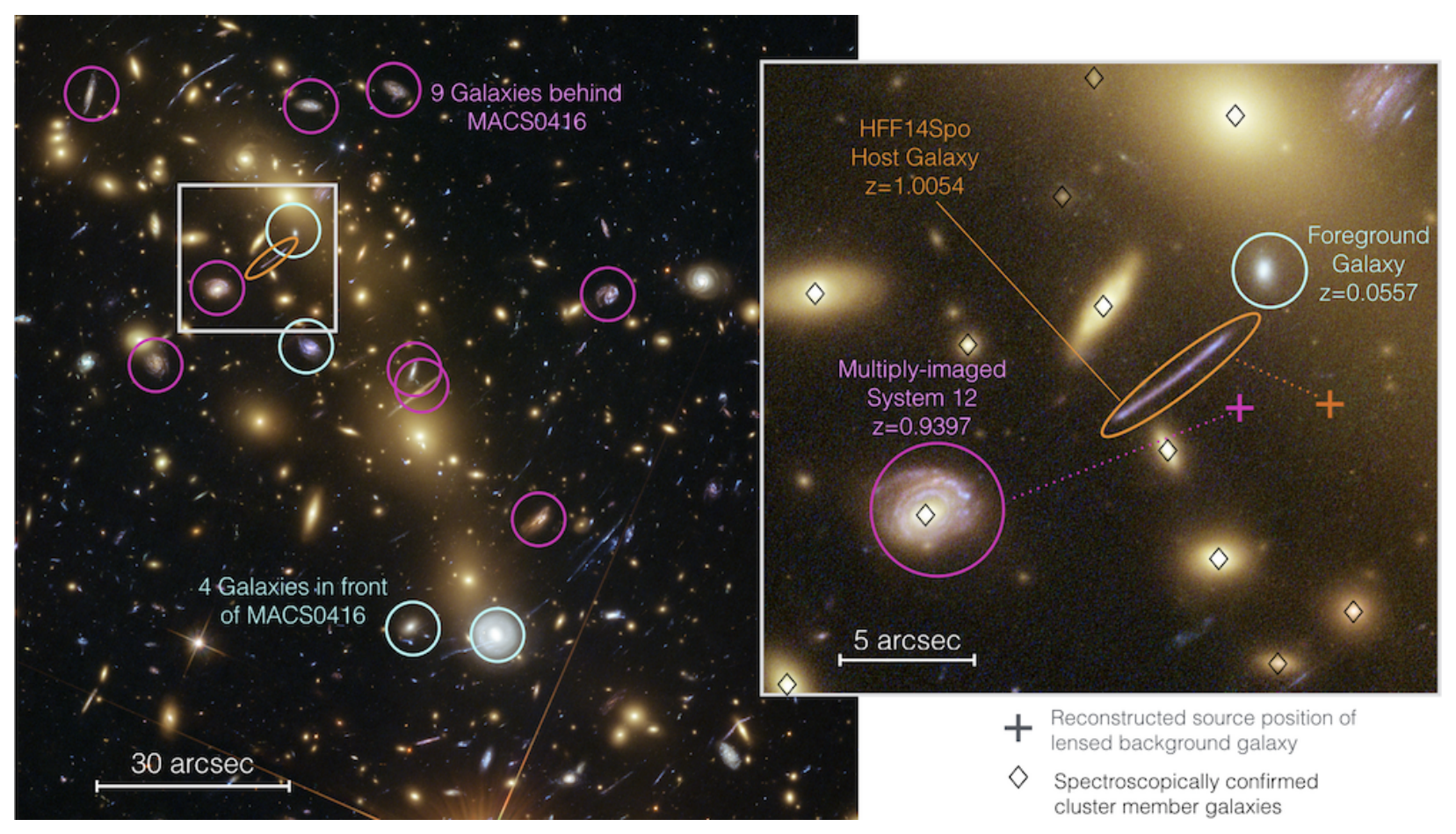

Supplementary Figure 2: Galaxies in the foreground and background of the MACS0416 cluster that are included in the GLEE-var model, which is a variation of the GLEE model that accommodates multi-plane lensing. The larger panel at left marks nine galaxies with spectroscopic redshifts greater than the cluster redshift (magenta circles) and four galaxies in the cluster foreground (light-blue circles). The inset panel at right zooms in on the HFF14Spo host galaxy (enclosed by the orange ellipse in each panel). Cluster member galaxies with spectroscopic redshifts that were included in the GLEE models are marked with black diamonds. The magenta circle marks a spiral galaxy at $z=0.9397$, which is also strongly lensed by the MACS0416 cluster into three highly distorted images (System 12 in [Ref. 1]). This image of the System 12 galaxy is further strongly lensed into arcs around a cluster member galaxy, which is marked by the black diamond near the center of the magenta circle. The galaxy in the foreground of the cluster at $z=0.0557$ is encircled in light blue. Crosses mark the reconstructed source positions (from the GLEE model) for the $z=0.9397$ galaxy and the HFF14Spo host. 
192 degrees of freedom $\left(\chi_{\nu}^{2}=1.7\right)$ and $\mathrm{AIC}=769$. This suggests that the multiple critical curve GLAFIC-var model is strongly disfavored by the positional strong-lensing constraints that are used for both models. However, we note that neither model incorporates the temporal constraints of the observed time delay.

A second variation of the CATS model (CATS-var2) was also used to test the plausibility of multiple critical curves intersecting the HFF14Spo locations. As in the GLAFIC-var case, this model requires that critical curves pass very near the HFF14Spo positions. The model can accommodate that constraint, insofar as the root mean square (RMS) error of the best-fit model is similar to that of the CATS and CATS-var models. However, in this CATS-var2 model the HFF14Spo host galaxy is predicted to be multiply-imaged 5 times. The HST images do not exhibit any breaks or substructure in the arc that would be generally expected in such a situation.

Moreover, this CATS-var2 model has strong implications for a separate background galaxy in the vicinity of image 11.3 (system 14 in [Ref. 1]). This galaxy is strongly lensed by a pair of spectroscopically confirmed cluster member galaxies ${ }^{1}$. Comparing the observed positions of the multiple images of System 14 against the CATS-var2 model-predicted positions, we find that this System contributes significantly to the global RMS error for the model-indicating that the CATS-var2 model can not accurately reproduce the multiple images of System 14. Conversely, when this system is removed as a model constraint, the RMS error decreases, and the CATS-var2 model can more successfully pass the critical line through the two HFF14Spo locations. A possible interpretation of this is that the established strong lensing constraints (especially System 14) are incompatible with the requirement that multiple critical curves must intersect the two HFF14Spo locations.

Linear Fits to Light Curve Data. Supplementary Figure 3 shows the linear interpolation used to define the rise and decline times for the HFF14Spo events, as described in Methods. Supplementary Figure 4 displays the color curves derived from these linear interpolations.

As shown in Supplementary Figure 3, the resulting piecewise linear fits are simplistic, but nevertheless approximately capture the observed behaviour for both events. Furthermore, since this toy model is not physically motivated, it allows us to remain agnostic for the time being as to the astrophysical source(s) driving these transients. From these fits we can see that HFF14SpoNW most likely reached a peak magnitude between 25 and $26.5 \mathrm{AB}$ mag in both F814W and $\mathrm{F} 435 \mathrm{~W}$, and had a decline timescale $t_{2}$ of less than 2 days in the rest frame. The observations of HFF14Spo-SE provide less stringent constraints, but we see that it had a peak between 23 and 26.5 $\mathrm{AB}$ mag in F160W and exhibited a decline time of $<7$ days. These fits also illustrate the generic fact that a higher peak brightness corresponds to a longer rise time and a faster decline timescale, independent of the specific model used. Changes to the arbitrary constraints we placed on these linear fits do not substantially affect these results.

Host Galaxy To examine whether the two transients originated from the same physical location in the source plane, we looked for differences in the properties of the HFF14Spo host galaxy at the 

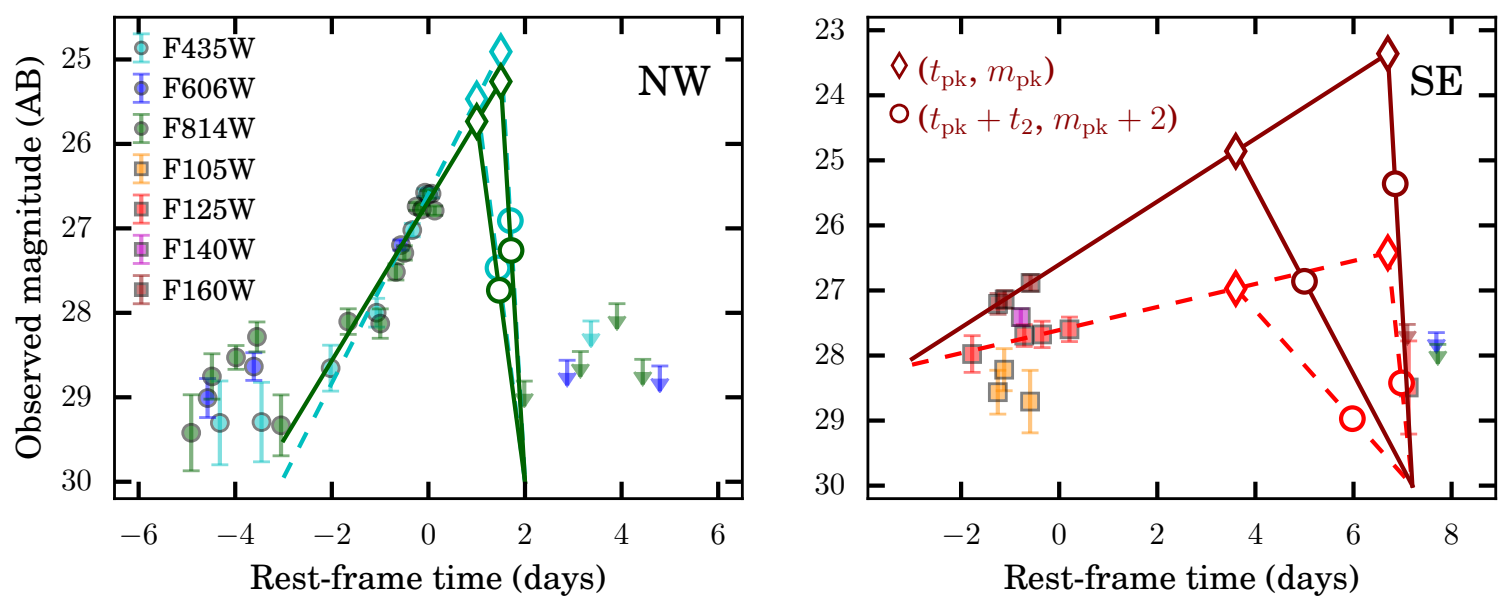

Supplementary Figure 3: Piecewise linear fits to the HFF14Spo light curves, used to measure the rise time and decay time of the two events. The HFF14Spo-NW light curve is shown in the top panel, and HFF14Spo-SE in the bottom. Filled points with error bars plot the observed brightness of each event in $\mathrm{AB}$ magnitudes as a function of rest-frame time (for $z=1.0054$ ). Piecewise linear fits are shown for the four bands that have enough points for fitting: in the top panel fits are plotted for the F814W band (solid green lines) and the F435W band (dashed cyan lines), while in the bottom panel fits are shown for F160W (solid maroon) and F125W (dashed scarlet). Open diamonds in each panel show three examples of assumptions for the time of peak brightness, $t_{\mathrm{pk}}$ (i.e., the position where the rising piece of the linear fit ends). Open circles mark the corresponding point, $t_{\mathrm{pk}}+t_{3}$, at which the fading transient would have declined in brightness by 3 mag. See text for details on the fitting procedure. 


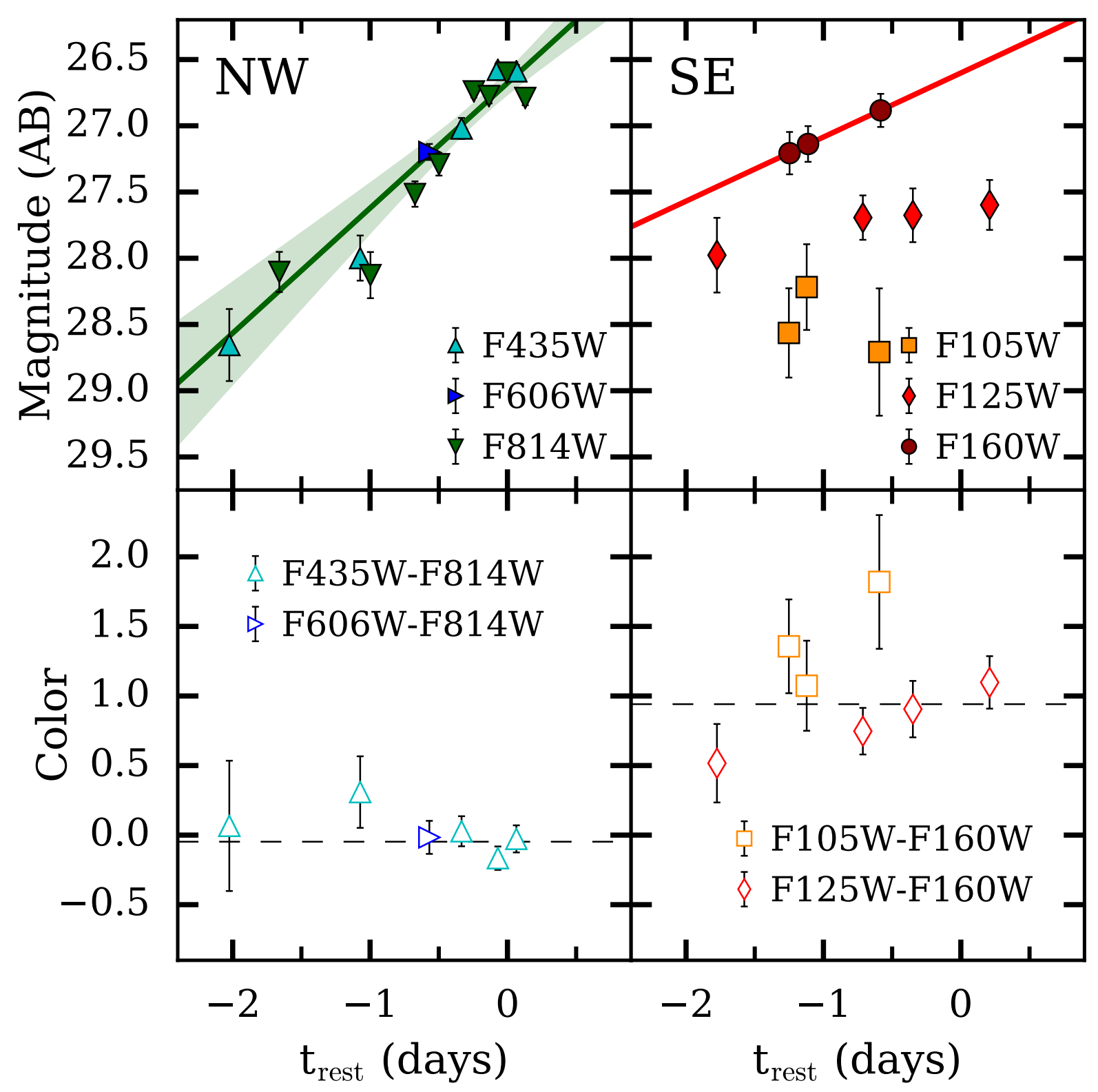

Supplementary Figure 4: Observed colors for the HFF14Spo events. Filled points plotted in the top panels show observed AB magnitudes for the HFF14Spo-NW and HFF14Spo-SE events from 3 rest-frame days before the date of observed peak brightness. Error bars represent $1 \sigma$ measurement uncertainties. Solid lines and shaded regions show linear fits to the data in the F814W and F160W bands. Open points in the bottom panels show observed colors in the rest-frame bands. For each color the magnitude in the bluer band is the directly measured value, and the magnitude in the redder band is interpolated from the linear fits shown in the top panel. Error bars in the lower panels represent $1 \sigma$ measurement uncertainties for the measured value only. All colors are consistent with showing no evolution over the rising portion of each light curve. 
Supplementary Table 1. Photometry of the HFF14Spo-NW event. The table here presents only the data in the F814W band. The full table of multi-band photometry is provided in machine-readable format as a separate supplementary file.

\begin{tabular}{|c|c|c|c|}
\hline $\begin{array}{l}\text { Date } \\
(\mathrm{MJD})\end{array}$ & Filter & $\begin{array}{c}\text { Flux Density } \\
\left(10^{30} \mathrm{erg} \mathrm{s}^{-1} \mathrm{~cm}^{-2} \mathrm{~Hz}^{-1}\right)\end{array}$ & AB Mag \\
\hline 56144.80 & F814W & $0.314 \pm 0.317$ & $<27.66$ \\
\hline 56157.83 & F814W & $0.028 \pm 0.041$ & $<30.29$ \\
\hline 56170.72 & F814W & $-0.108 \pm 0.109$ & $<27.61$ \\
\hline 56184.75 & F814W & $0.004 \pm 0.007$ & $<32.47$ \\
\hline 56662.85 & F814W & $0.062 \pm 0.026$ & $29.42 \pm 0.45$ \\
\hline 56663.71 & F814W & $0.114 \pm 0.028$ & $28.75 \pm 0.27$ \\
\hline 56664.71 & F814W & $0.141 \pm 0.018$ & $28.53 \pm 0.14$ \\
\hline 56665.58 & F814W & $0.176 \pm 0.029$ & $28.28 \pm 0.18$ \\
\hline 56666.58 & F814W & $0.067 \pm 0.022$ & $29.33 \pm 0.36$ \\
\hline 56669.37 & F814W & $0.208 \pm 0.029$ & $28.10 \pm 0.15$ \\
\hline 56670.70 & F814W & $0.204 \pm 0.033$ & $28.13 \pm 0.17$ \\
\hline 56671.35 & F814W & $0.358 \pm 0.032$ & $27.52 \pm 0.10$ \\
\hline 56671.70 & F814W & $0.440 \pm 0.034$ & $27.29 \pm 0.09$ \\
\hline 56672.21 & F814W & $0.731 \pm 0.031$ & $26.74 \pm 0.05$ \\
\hline 56672.43 & F814W & $0.707 \pm 0.036$ & $26.78 \pm 0.06$ \\
\hline 56672.69 & F814W & $0.832 \pm 0.035$ & $26.60 \pm 0.05$ \\
\hline 56672.96 & F814W & $0.698 \pm 0.035$ & $26.79 \pm 0.06$ \\
\hline 56676.68 & F814W & $0.019 \pm 0.036$ & $<30.68$ \\
\hline 56679.01 & F814W & $-0.015 \pm 0.050$ & $<28.46$ \\
\hline 56680.53 & F814W & $-0.109 \pm 0.084$ & $<27.89$ \\
\hline 56681.60 & F814W & $-0.021 \pm 0.046$ & $<28.55$ \\
\hline 56686.36 & F814W & $-0.053 \pm 0.068$ & $<28.12$ \\
\hline 56686.64 & F814W & $-0.092 \pm 0.077$ & $<27.99$ \\
\hline 56691.36 & F814W & $-0.074 \pm 0.074$ & $<28.03$ \\
\hline 56697.73 & F814W & $-0.040 \pm 0.051$ & $<28.43$ \\
\hline 56916.98 & F814W & $0.007 \pm 0.093$ & $<31.86$ \\
\hline
\end{tabular}


Supplementary Table 2. Photometry of the HFF14Spo-SE event. The table here presents only the data in the F160W band. The full table of multi-band photometry is provided in machine-readable format as a separate supplementary file.

\begin{tabular}{clcc}
\hline \hline $\begin{array}{c}\text { Date } \\
(\mathrm{MJD})\end{array}$ & Filter & $\begin{array}{c}\text { Flux Density } \\
\left(10^{30} \mathrm{erg} \mathrm{s}^{-1} \mathrm{~cm}^{-2} \mathrm{~Hz}^{-1}\right)\end{array}$ & AB Mag \\
\hline 56132.24 & F160W & $-0.196 \pm 0.355$ & $<26.33$ \\
56144.87 & F160W & $-0.592 \pm 0.716$ & $<25.57$ \\
56170.79 & F160W & $-0.375 \pm 0.461$ & $<26.05$ \\
56197.79 & F160W & $-0.766 \pm 0.725$ & $<25.56$ \\
56689.36 & F160W & $-0.282 \pm 0.405$ & $<26.19$ \\
56870.00 & F160W & $0.028 \pm 0.069$ & $<30.30$ \\
56870.99 & F160W & $0.162 \pm 0.059$ & $28.38 \pm 0.39$ \\
56877.70 & F160W & $-0.047 \pm 0.131$ & $<27.42$ \\
56877.96 & F160W & $-0.002 \pm 0.095$ & $<27.76$ \\
56879.69 & F160W & $0.004 \pm 0.086$ & $<32.34$ \\
56880.61 & F160W & $-0.043 \pm 0.113$ & $<27.57$ \\
56880.88 & F160W & $0.126 \pm 0.065$ & $<28.65$ \\
56881.94 & F160W & $-0.026 \pm 0.125$ & $<27.46$ \\
56890.04 & F160W & $0.035 \pm 0.078$ & $<30.05$ \\
56899.00 & F160W & $0.476 \pm 0.070$ & $27.21 \pm 0.16$ \\
56899.27 & F160W & $0.507 \pm 0.063$ & $27.14 \pm 0.13$ \\
56900.33 & F160W & $0.640 \pm 0.074$ & $26.88 \pm 0.12$ \\
56915.73 & F160W & $0.053 \pm 0.119$ & $<29.59$ \\
56922.41 & F160W & $0.037 \pm 0.235$ & $<29.99$ \\
56928.13 & F160W & $-0.281 \pm 0.466$ & $<26.04$ \\
\hline & & & \\
\hline
\end{tabular}


Supplementary Table 3. Properties of the local stellar population in the HFF14Spo host galaxy, from SED fitting.

\begin{tabular}{lccc}
\hline \hline $\begin{array}{l}\text { Host image: } \\
\text { Location: }\end{array}$ & $\begin{array}{c}11.1 \\
\text { HFF14Spo-SE }\end{array}$ & $\begin{array}{c}11.2 \\
\text { HFF14Spo-NW }\end{array}$ & $\begin{array}{c}11.3 \\
\text { center }\end{array}$ \\
\hline$(U-V)_{\text {rest }}$ & $0.69_{-0.05}^{+0.2}$ & $0.52_{-0.10}^{+0.15}$ & $0.39 \pm 0.05$ \\
$\log \left[\Sigma\left(M_{*} / \mathrm{M}_{\odot}\right)\right]$ & $7.14 \pm 0.15$ & $7.14 \pm 0.15$ & $7.04 \pm 0.10$ \\
$\operatorname{Age}(\mathrm{Gyr})$ & $0.292 \pm 0.5$ & $0.290 \pm 0.5$ & $0.292 \pm 0.5$ \\
\hline
\end{tabular}

location of each event. We first used the technique of "pixel-by-pixel" SED fitting ${ }^{4}$ to determine rest-frame colors and stellar properties in a single resolution element of the HST imaging data. For this purpose we used the deepest possible stacks of $H S T$ images, comprising all available data except those images where the transient events were present. The resulting maps of stellar population properties are shown in Supplementary Figure 5. Supplementary Table 3 reports measurements of the three derived stellar population properties (color, mass, age) from host images 11.1, 11.2 and 11.3. In 11.1 and 11.2 these measurements were extracted from the central pixel at the location of each of the two HFF14Spo events. The lensing magnification here ranges from $\mu=10$ to 200 , corresponding to a size on the source plane between 6 and $600 \mathrm{pc}^{2}$. For host image 11.3 we report the stellar population properties derived from the pixel at the center of the galaxy, because the lens models do not have sufficient precision to map the HFF14Spo locations to specific positions in image 11.3. With a magnification of $\sim 3$ to 5 , this extraction region covers roughly 2000 to 6000 $\mathrm{pc}^{2}$.

The reported uncertainties for these derived stellar properties in Table 3 reflect only the measurement errors from the SED fitting, and do not attempt to quantify potential systematic biases. Such biases could arise, for example, from color differences in the background light, which is dominated by the cluster galaxies and varies significantly across the MACS0416 field. Such a bias might shift the absolute values of the parameter scales for any given host image (e.g., making the galaxy as a whole appear bluer, more massive, and younger). However, the gradients across any single host image are unlikely to be driven primarily by such systematics.

Supplementary Figure 5 and Supplementary Table 3 show that the measured values of the color, stellar mass, and age at the two HFF14Spo locations are mutually consistent. Thus, it is plausible to assume that the two positions map back to the same physical location at the source plane. Comparing those two locations to the center of the galaxy as defined in image 11.3, we see only a mild tension in the rest frame $U-V$ color. This comparison therefore cannot quantitatively rule out the possibility that the two transient events are located at the center of the galaxy. However, the maps shown in Supplementary Figure 5 do show a gradient in both $U-V$ color and stellar age. For both images 11.1 and 11.2 the bluest and youngest stars $(U-V \approx 0.3, \tau \approx 280 \mathrm{Myr})$ 

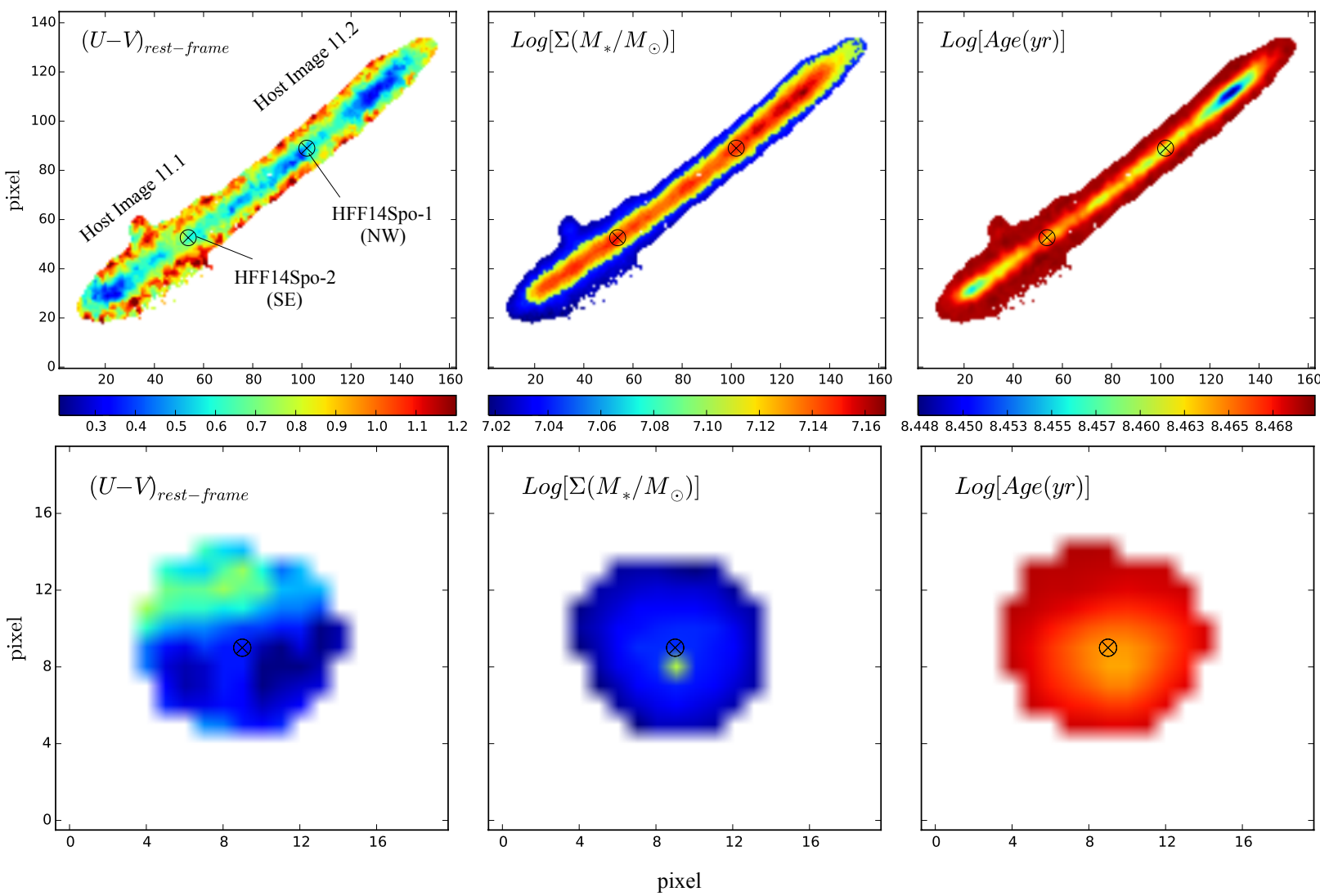

Supplementary Figure 5: Stellar population properties of the HFF14Spo host galaxy, derived from "pixel-by-pixel" SED fitting. The top row shows maps for the adjacent host images 11.1 and 11.2, and the bottom panels show image 11.3. From left to right the panels present the rest-frame $(U-V)$ color, the stellar surface mass density $\Sigma$, and the mean age of the stellar population in Gyr. Markers in the top row denote the positions of the two HFF14Spo transient events. Markers in the bottom panels are at the center of host image 11.3. 
Supplementary Table 4: Measurements of the [OII] $\lambda \lambda 3626,3629$ lines from HFF14Spo host galaxy images 11.1 and 11.2

\begin{tabular}{|c|c|c|c|c|c|c|c|c|c|c|}
\hline \multirow{2}{*}{$\begin{array}{c}\text { Aperture } \\
\text { ID }\end{array}$} & \multirow{2}{*}{$\begin{array}{c}\text { R.A. } \\
\text { J2000 } \\
\text { (degrees) }\end{array}$} & \multirow{2}{*}{$\begin{array}{c}\text { Dec. } \\
\text { J2000 } \\
\text { (degrees) }\end{array}$} & \multirow{2}{*}{$\begin{array}{l}\text { distance to } \\
\text { HFF14Spo-SE } \\
\text { (Arcsec) }\end{array}$} & \multicolumn{3}{|c|}{$[\mathrm{OII}] \lambda 3726$} & \multicolumn{3}{|c|}{ [OOII] $\lambda 3729$} & \multirow{2}{*}{$\begin{array}{l}\text { Line } \\
\text { Ratio }\end{array}$} \\
\hline & & & & $\begin{array}{c}\text { Flux } \\
\left(\mathrm{erg} \mathrm{s}^{-1} \mathrm{~cm}^{-2}\right)\end{array}$ & $\begin{array}{c}\lambda_{\text {center }} \\
(\AA)\end{array}$ & $\begin{array}{c}\text { FWHM } \\
(\AA)\end{array}$ & $\begin{array}{c}\text { Flux } \\
\left(\mathrm{erg} \mathrm{s}^{-1} \mathrm{~cm}^{-2}\right)\end{array}$ & $\begin{array}{c}\lambda_{\text {center }} \\
(\AA)\end{array}$ & $\begin{array}{l}\text { FWHM } \\
(\AA)\end{array}$ & \\
\hline 1 & 64.039371 & -24.070450 & -1.54 & $2.19 \mathrm{e}-18$ & 7472.37 & 4.00 & $3.57 \mathrm{e}-18$ & 7478.17 & 4.00 & 1.63 \\
\hline 2 & 64.039218 & -24.070345 & -0.88 & $4.73 \mathrm{e}-18$ & 7472.16 & 4.00 & $5.30 \mathrm{e}-18$ & 7478.12 & 3.40 & 1.12 \\
\hline 3 & 64.039078 & -24.070264 & -0.30 & $5.05 \mathrm{e}-18$ & 7472.29 & 4.00 & $6.10 \mathrm{e}-18$ & 7478.27 & 3.73 & 1.21 \\
\hline 4 & 64.038921 & -24.070163 & 0.39 & $4.22 \mathrm{e}-18$ & 7472.19 & 4.00 & $5.74 \mathrm{e}-18$ & 7478.08 & 3.59 & 1.36 \\
\hline 5 & 64.038785 & -24.070078 & 0.97 & $3.86 \mathrm{e}-18$ & 7472.25 & 4.00 & $6.56 \mathrm{e}-18$ & 7478.19 & 4.00 & 1.70 \\
\hline 6 & 64.038637 & -24.069958 & 1.65 & $4.80 \mathrm{e}-18$ & 7472.51 & 4.00 & $5.42 \mathrm{e}-18$ & 7478.07 & 2.69 & 1.13 \\
\hline 7 & 64.038501 & -24.069865 & 2.24 & $4.60 \mathrm{e}-18$ & 7472.57 & 3.43 & $5.74 \mathrm{e}-18$ & 7478.17 & 3.20 & 1.25 \\
\hline 8 & 64.038352 & -24.069752 & 2.92 & $4.70 \mathrm{e}-18$ & 7472.54 & 3.54 & $6.22 \mathrm{e}-18$ & 7478.16 & 2.95 & 1.32 \\
\hline 9 & 64.038229 & -24.069648 & 3.50 & $3.26 \mathrm{e}-18$ & 7472.83 & 2.80 & $5.79 \mathrm{e}-18$ & 7478.16 & 2.84 & 1.77 \\
\hline 10 & 64.038076 & -24.069532 & 4.19 & $2.44 \mathrm{e}-18$ & 7473.01 & 2.57 & $3.22 \mathrm{e}-18$ & 7478.10 & 2.73 & 1.32 \\
\hline NW & 64.038565 & -24.069939 & 1.90 & $4.30 \mathrm{e}-18$ & 7472.55 & 3.13 & $5.49 \mathrm{e}-18$ & 7478.01 & 2.89 & 1.28 \\
\hline SE & 64.038998 & -24.070241 & 0.00 & $4.37 \mathrm{e}-18$ & 7472.46 & 4.00 & $6.10 \mathrm{e}-18$ & 7478.22 & 3.79 & 1.40 \\
\hline
\end{tabular}

are localized in knots near the extreme ends of each image, well separated from either of the HFF14Spo transient events. In the less distorted host image 11.3 the bluer and younger stars are concentrated near the center. Taken together, these color and age gradients suggest that the two transients are not coincident with the center of their host galaxy.

In addition to the HST imaging data, we also have spatially resolved spectroscopy from the MUSE integral field data. The only significant spectral line feature for the HFF14Spo host is the [OII] $\lambda \lambda$ 3726, 3729 doublet, observed at 7474 and $7478 \AA$. To examine this feature in detail, one-dimensional spectra were extracted from the three-dimensional MUSE data cube at a series of locations along the HFF14Spo host-galaxy arc.

At each position the lines were extracted using apertures with a radius of $0.6^{\prime \prime}$, so adjacent extractions are not independent, although the two extractions centered on the HFF14Spo-NW and -SE positions have no overlap. Supplementary Figure 6 depicts these extraction apertures, shows the observed [OII] lines at the HFF14Spo-NW and SE positions, and compares the [OII] line profiles to other positions along the length of the host-galaxy arc.

A difference in the shape of the [OII] lines or the doublet line ratio could provide evidence for a different environment at the two HFF14Spo locaions, which would suggest that the two events emerged from independent sources. For a visual test for spectral deviations, we first constructed a mean spectrum by averaging the 1-D spectra from five non-overlapping apertures (apertures 1, $3,5,7,9)$. To account for differences in magnification and host-galaxy intensity across the arc, each input spectrum was normalized at the wavelength $7477.7 \AA$, which corresponds to the center of the $\lambda 3729$ component of the [OII] emission line. This mean spectrum was then subtracted from the 1-D spectrum of each aperture, producing a set of "residual spectra," shown in Supplementary Figure 6 in the lower-left panel. These spectra show no indication of a systematic trend in the wavelength position, shape or line ratio across the arc. Similarly, a comparison of the spectra 

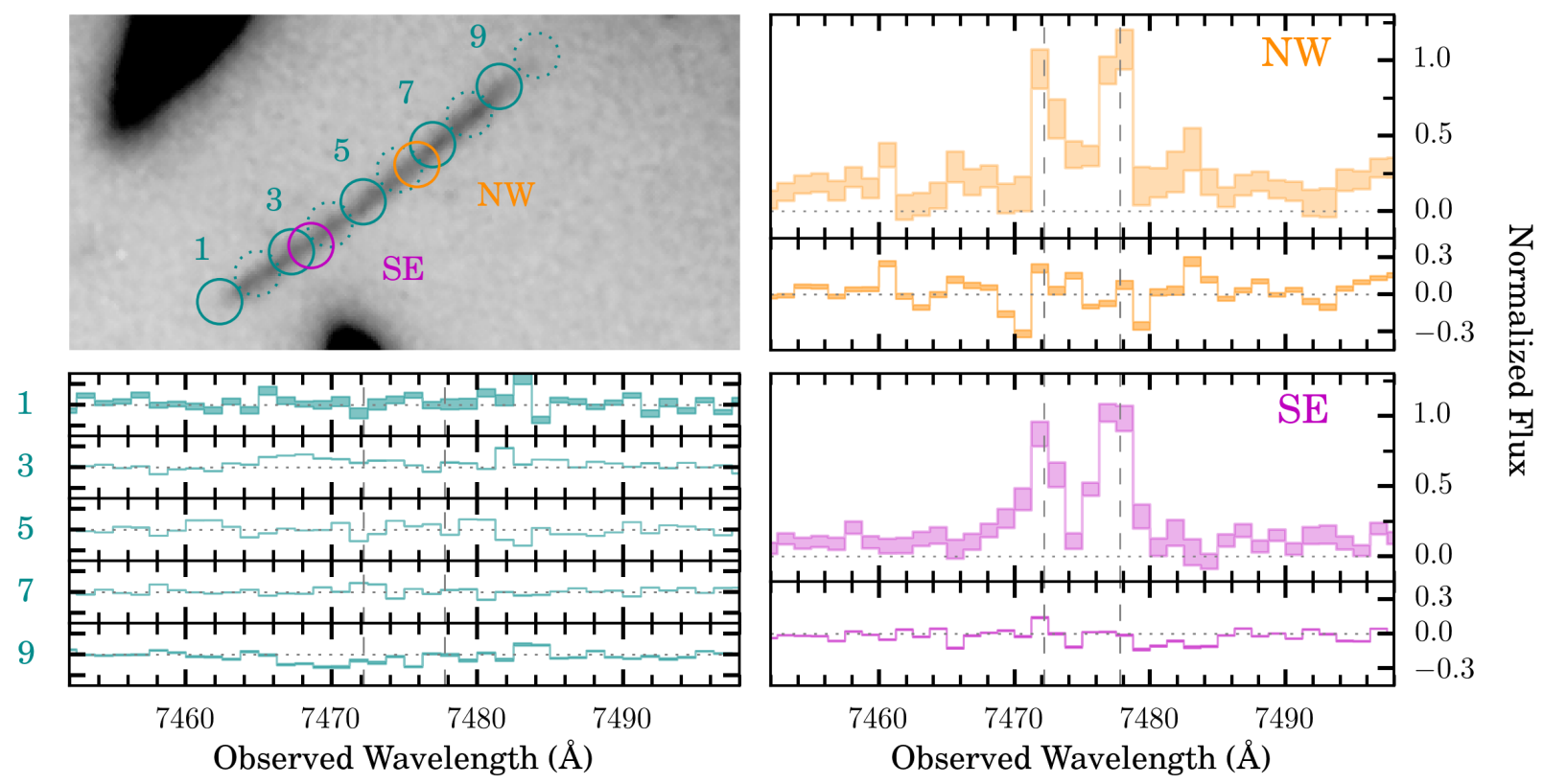

Supplementary Figure 6: Measurements of the [OII] $\lambda \lambda 3726,3729$ doublet, observed with MUSE after both HFF14Spo events had faded. The upper-left panel shows the 12 apertures with radius $0.6^{\prime \prime}$ that were used for the extractions reported in Table 4. Odd-numbered apertures are plotted with solid lines, while even-numbered apertures are shown as unlabeled dashed circles. The apertures centered on the HFF14Spo-NW and SE locations are highlighted in orange and magenta, respectively. The 1-D spectra extracted from these HFF14Spo locations are shown in the upperright and lower-right panels, centered around the observed wavelength of the [OII] doublet, and normalized to reach a value of unity at the peak of the $\lambda 3729$ line. Dashed vertical lines mark the vacuum wavelengths of the doublet, redshifted to $z=1.0054$. Dotted horizontal lines mark zero flux. The width of the shaded region indicates the $1 \sigma$ uncertainty in the measured flux. Below each spectrum, a residual plot shows the flux that remains after subtracting off a mean spectrum constructed from the normalized spectra of the odd-numbered apertures. The lower-left panel shows the same residual spectra constructed for the odd-numbered apertures, demonstrating that the [OII] line profile does not exhibit any significant gradients across the length of the host-galaxy arc. 
from the HFF14Spo-NW and SE locations (right panels of Supplementary Figure 6) reveals no significant difference in the [OII] line shapes.

This qualitative comparison is borne out by a more quantitative assessment, reported in Supplementary Table 4. We fit a Gaussian profile to each component of the [OII] doublet, separately in each extracted 1-D spectrum. From these fits we measured the integrated line flux, observed wavelength of line center $\left(\lambda_{\text {center }}\right)$, full width at half maximum intensity (FWHM), and the intensity ratio of the two components of the doublet. These quantities - all reported in Supplementary Table 4-do not exhibit any discernible gradient across the host galaxy. Thus, the [OII] measurements from MUSE cannot be used to distinguish either HFF14Spo location from the other, or to definitively answer whether either position is coincident with the center of the host galaxy (as would be required, for example, if these transients were from an AGN). We conclude that it is entirely plausible but not certain that the two HFF14Spo events arose from the same physical location in the host galaxy.

LBV Light-Curve Comparison. Supplementary Figure 7 presents a direct comparison of the observed HFF14Spo light curves against the light curves of the two LBVs that have well-studied rapid eruptions: SN 2009ip and NGC3432-LBV1. The brief outbursts of these LBVs have been less finely sampled than the two HFF14Spo events, but the available data show a wide variety of rise and decline times, even for a single object over a relatively narrow time window of a few months.

RN Light-Curve Comparison. There are ten known RNe in the Milky Way galaxy, and seven of these exhibit outbursts that decline rapidly, fading by two magnitudes in less than ten days ${ }^{5}$. Supplementary Figure 8 compares the HFF14Spo light curves to a composite light curve (the gray shaded region), which encompasses the $\mathrm{V}$ band light curve templates ${ }^{5}$ for all seven of these galactic $\mathrm{RN}$ events. The Andromeda galaxy (M31) also hosts at least one RN with a rapidly declining light curve. The 2014 eruption of this well-studied nova, M31N 2008-12a, is shown as a solid black line in Supplementary Figure 8 , fading by 2 mag in $<3$ days. This comparison demonstrates that the rapid decline of both of the HFF14Spo transient events is fully consistent with the eruptions of known RNe in the local universe.

RN Luminosity and Recurrence Period. Supplementary Figure 9 plots first the RN outburst amplitude (the apparent magnitude between outbursts minus the apparent magnitude at peak) and then the peak luminosity against the log of the recurrence period in years. For the HFF14Spo events we can only measure a lower limit on the outburst amplitude, since the presumed progenitor star is unresolved, so no measurement is available at quiescence. Supplementary Figure 9 shows that a recurrence period as fast as one year is expected only for a RN system in which the primary white dwarf is both very close to the Chandrasekhar mass limit $\left(1.4 \mathrm{M}_{\odot}\right)$ and also has an extraordinarily rapid mass transfer rate $\left(\sim 10^{-6} \mathrm{M}_{\odot} \mathrm{yr}^{-1}\right)$. The models of [Ref. 7] suggest that such systems should have a very low peak amplitude (barely consistent with the lower limit for HFF14Spo) and a low peak luminosity ( $\sim 100$ times less luminous than the HFF14Spo events). 

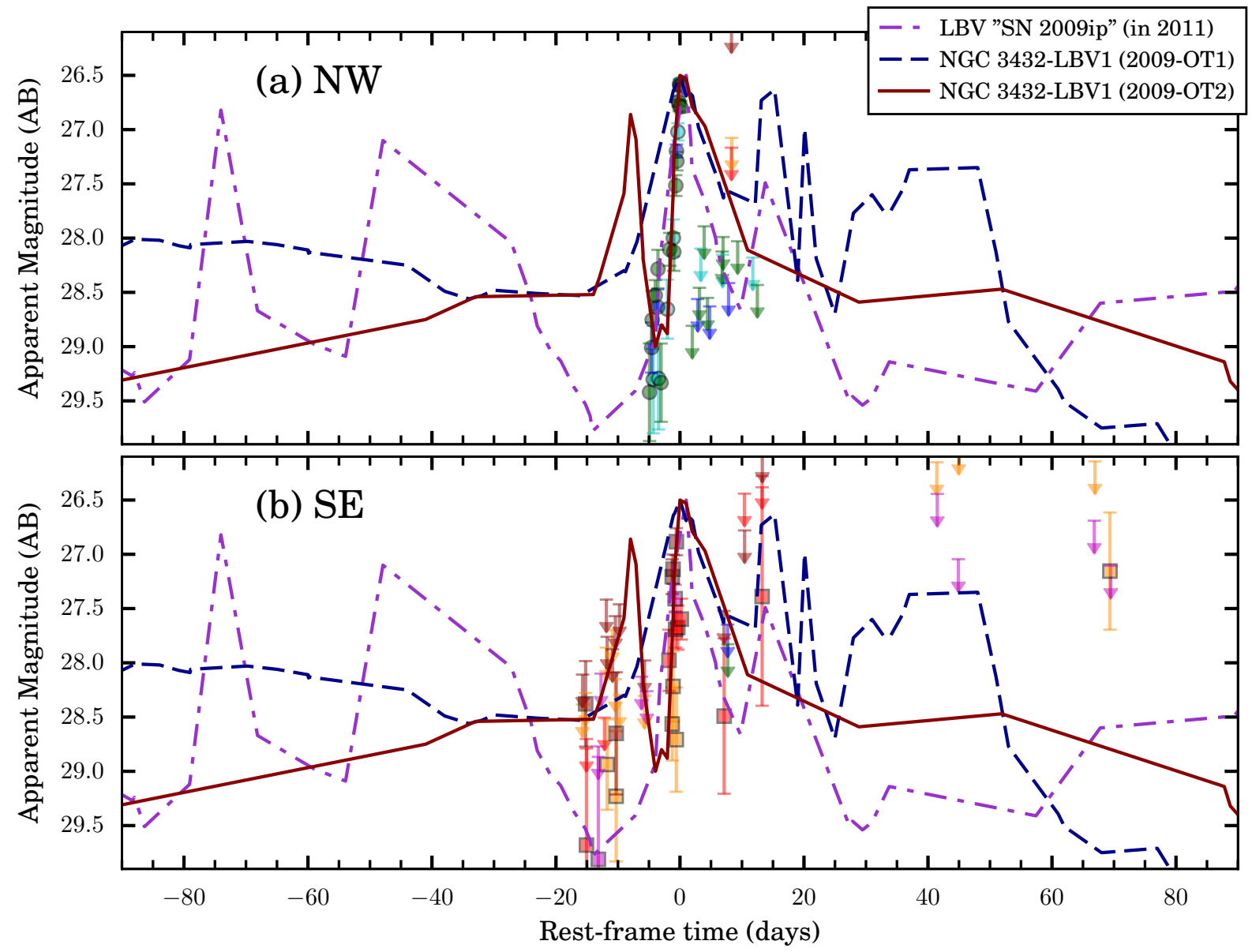

Supplementary Figure 7: Comparison of observed HFF14Spo light curves against rapid outbursts from two LBVs. Colored markers show the HFF14Spo light-curve data, as in Figure 4 in the main text, with downward arrows marking $3 \sigma$ upper limits in epochs with no detection of the HFF14Spo source. The LBV comparison light curves have been shifted in time and magnitude to match up with the peak of the observed light curves. 


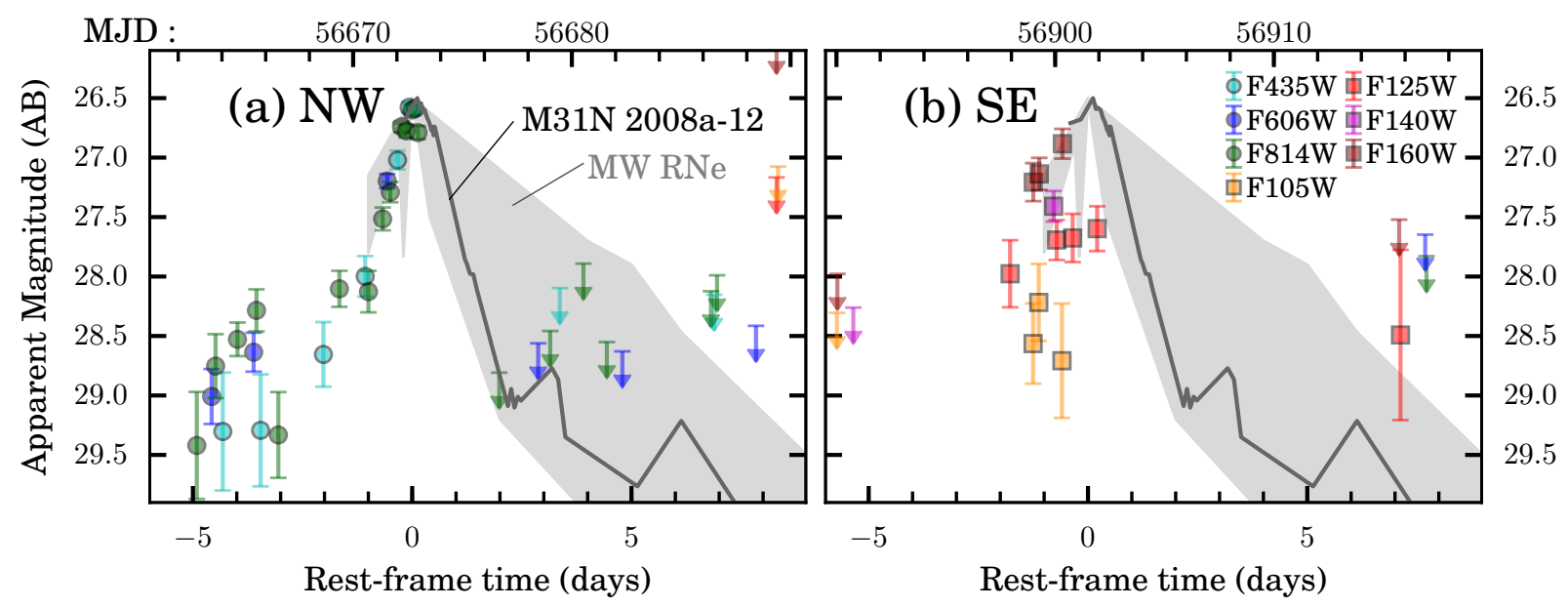

Supplementary Figure 8: Comparison of the HFF14Spo light curves against template light curves for RN outbursts. Colored markers show the HFF14Spo light-curve data, as in Figure 4 in the main text, plotting the apparent magnitude as a function of time in the rest frame (bottom axis) and observer frame (top axis). The gray shaded regions encompass the outburst light-curve shapes of 5 of the 11 known Galactic RNe (U Sco, V2487 Oph, V394 CrA, T CrB, and V745 Sco), selected because they exhibit a rapid decline in their light curves ${ }^{5}$. The solid black line traces the 2014 outburst light-curve shape for the rapid-recurrence nova M31N 2008a-12 6 . All template light curves have been normalized to match the observed peaks of the HFF14Spo events. 

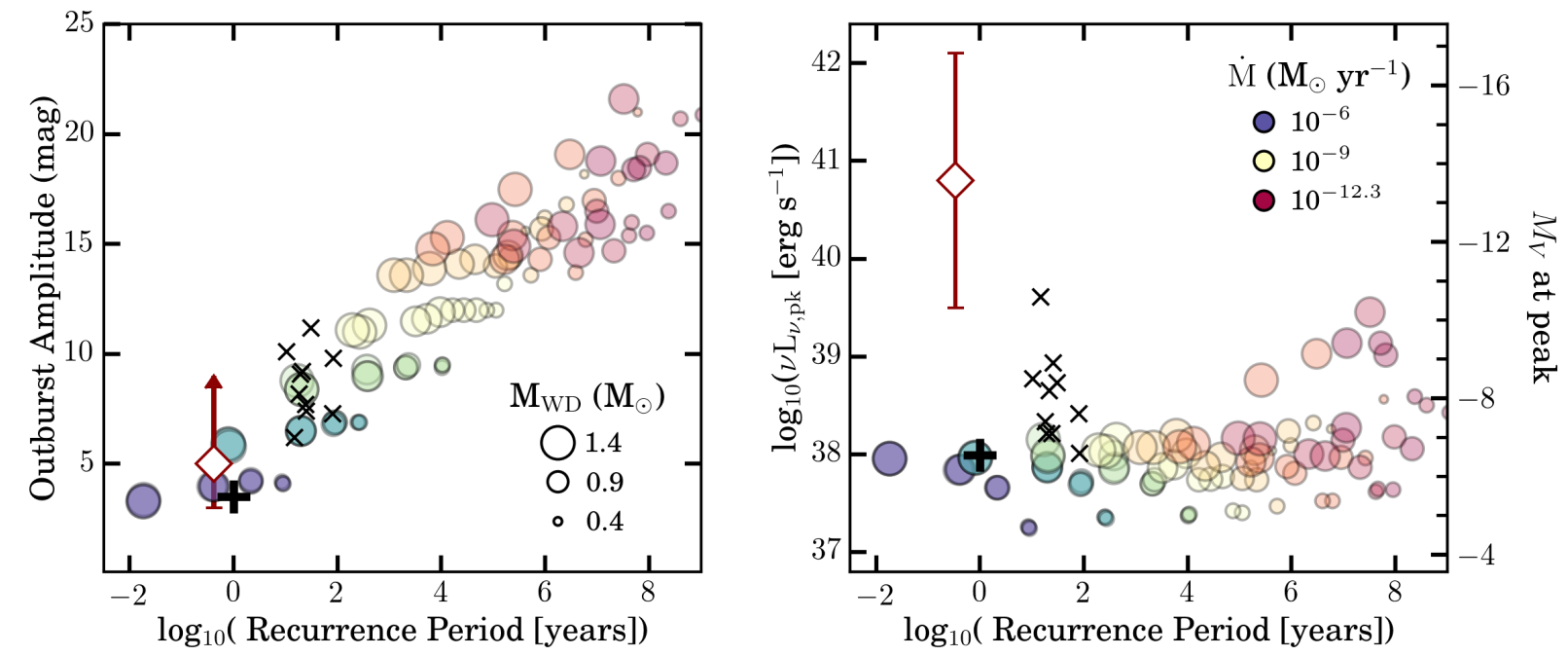

Supplementary Figure 9: Comparison of the inferred HFF14Spo recurrence timescale against observed $\mathrm{RNe}$ and models. In the left panel the outburst amplitude in magnitudes is plotted against the recurrence timescale, while in the right panel the ordinate axis shows the peak luminosity (or absolute magnitude). In both panels, the joint constraints on HFF14Spo from both transient episodes are plotted as large open diamonds, with error bars indicating the uncertainty due to magnification and interpolation of the light curve. Observed constraints from the 10 Galactic RNe appear as black " $x$ " points, and the rapid-recurrence nova M31N 2008a-12 is shown with a thick black "+" marker. Uncertainties on these values are not shown, but are typically of order 0.1 mags, smaller than the size of the points. Colored circles show the results from a suite of numerical hydrodynamic simulations ${ }^{7}$. The size of each circle indicates the mass of the primary white dwarf (WD) star over the range $0.4-1.4 \mathrm{M}_{\odot}$, as indicated in the legend of the left panel. The color of each circle denotes the rate of mass transfer from the secondary onto the WD, as given in the right panel's legend. 
The closest analog for the HFF14Spo events from the population of known RN systems is the nova M31N2008-12a. [Ref. 8] provided a theoretical model that can account for the key observational characteristics of this remarkable nova: the very rapid recurrence timescale $(<1 \mathrm{yr})$, fast optical light curve ( $t_{2} \sim 2$ days), and short supersoft x-ray phase (6-18 days after optical outburst $)^{9}$. To match these observations, [Ref. 8 ] invoke a $1.38 \mathrm{M}_{\odot}$ white dwarf primary, drawing mass from a companion at a rate of $1.6 \times 10^{-7} \mathrm{M}_{\odot} \mathrm{yr}^{-1}$. This is largely consistent with the theoretical expectations derived by [Ref. 7], and reinforces the conclusion that a combination of a high-mass white dwarf and efficient mass transfer are the key ingredients for rapid recurrence and short light curves. However, the peculiarly high luminosity of the HFF14Spo events cannot be effectively explained with this hypothesis, even after accounting for the very large uncertainties.

Expected Timescale for Microlensing Events. A commonly observed example of microlensinginduced transient effects is when a bright background source (a quasar) is magnified by a galaxyscale lens ${ }^{10 \mid 11}$. In this optically thick microlensing regime, the lensing potential along the line of sight to the quasar is composed of many stellar-mass objects. Each compact object along the line of sight generates a separate critical lensing curve, resulting in a complex web of overlapping critical curves. As all of these lensing stars are in motion relative to the background source, the web of caustics will shift across the source position, leading to a stochastic variability on timescales of months to years. This scenario is inconsistent with the observed data, as the two HFF14Spo events were far too short in duration and did not exhibit the repeated "flickering" variation that would be expected from optically thick microlensing.

For the cluster-scale lens relevant in the case of HFF14Spo, we should expect to be in the optically thin microlensing regime. This situation is similar to the "local" microlensing light curves observed when stars within our Galaxy or neighboring dwarf galaxies pass behind a massive compact halo object $12-15$. In this case, an isolated microlensing event can occur if there is a background star (i.e., in the HFF14Spo host galaxy) that is the dominant source of luminosity in its environment. In practice this means that the source must be a very bright $\mathrm{O}$ or $\mathrm{B}$ star with mass of order $10 \mathrm{M}_{\odot}$. Depending on its age, the size of such a star would range from a few to a few dozen times the size of the Sun. The net relative transverse velocity would be on the order of a few $100 \mathrm{~km} \mathrm{~s}^{-1}$, which is comparable to the orbital velocity of stars within a galaxy or galaxies within a cluster.

In the case of a smooth cluster potential, the timescale $\tau$ for the light curve of such a caustic crossing event is dictated by the radius of the source, $R$, and the net transverse velocity, $v$, of the source across the caustic ${ }^{16-18}$ as

$$
\tau=\frac{6 R}{5 \mathrm{R}_{\odot}} \frac{300 \mathrm{~km} \mathrm{~s}^{-1}}{v} \mathrm{hr} .
$$

Thus, for reasonable assumptions about the star's radius and velocity, the timescale $\tau$ is on the order of hours to days, which is well matched to the observed rise and decline timescales of the HFF14Spo events. 


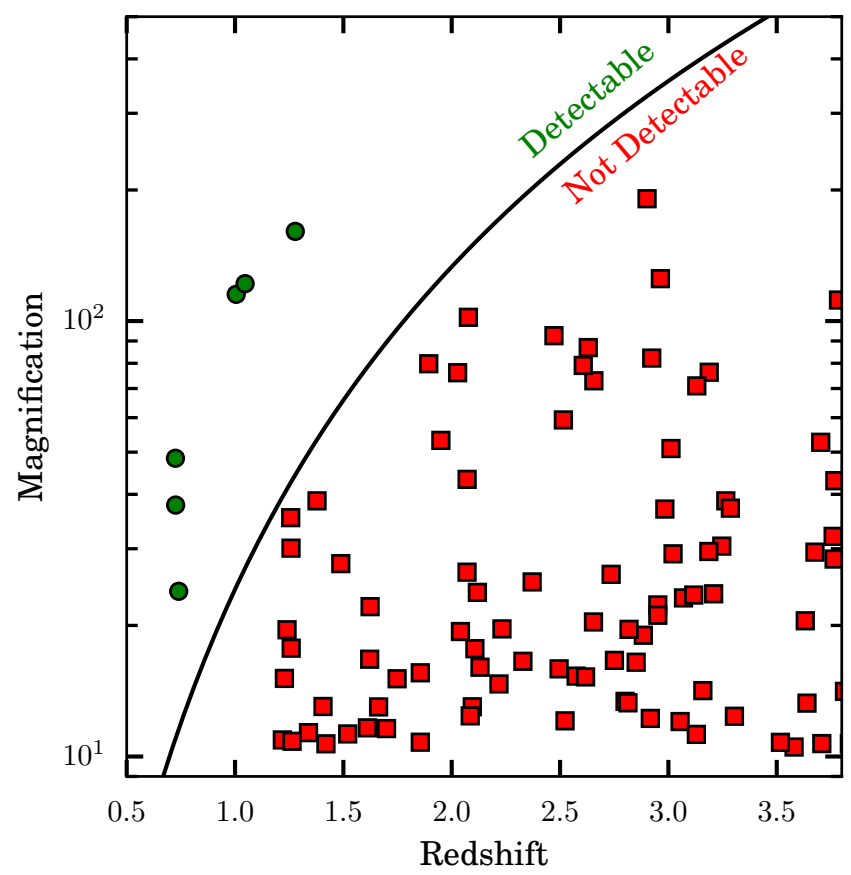

Supplementary Figure 10: Model-predicted magnification and redshift for strongly lensed galaxies behind all six of the HFF clusters (Abell 2744, Abell 370, Abell S1063, MACS J1149, MACS J0416, and MACS J0717). The black curve shows the minimum magnification required to make a HFF14Spo-like transient detectable, as a function of redshift. To define the distance modulus as a function of redshift for this curve, a standard $\Lambda$ CDM cosmological model is adopted 19 . Green circles above the line represent the six galaxies in which a transient with $M_{V}>-14$ mag would be sufficiently magnified, and red squares below the line mark all the other galaxies in which the HFF14Spo events would not have been detected.

Detectability of Similar Events in Other Lensed Galaxies. Supplementary Figure 10 shows the known strongly-lensed galaxies in the Hubble Frontier Fields for which a HFF14Spo-like transient could be detected, based on each galaxy's redshift and model-predicted magnification.

As described in Methods, the small number of galaxies with similar magnification allows for the possibility that the intrinsic rate of HFF14Spo-like transients is as high as $R=1.5$ events galaxy $^{-1} \mathrm{yr}^{-1}$. Future examination of the rate of such transients should consider the total stellar mass and the star-formation rates of the galaxies surveyed, or use a projection of the lensed background area onto the source plane to derive a volumetric rate. Such analyses would require a more detailed exploration of the impact of lensing uncertainties on derived properties of the lensed galaxies and the lensed volume in the high-magnification regions, and this is beyond the scope of the current work. 


\section{References}

1. Caminha, G. B. et al. A refined mass distribution of the cluster MACS J0416.1-2403 from a new large set of spectroscopic multiply lensed sources. A\&A 600, A90 (2017).

2. Sérsic, J. L. Influence of the atmospheric and instrumental dispersion on the brightness distribution in a galaxy. Boletin de la Asociacion Argentina de Astronomia La Plata Argentina 6 , 41 (1963).

3. Akaike, H. A new look at the statistical model identification. Automatic Control, IEEE Transactions on 19, 716-723 (1974).

4. Hemmati, S. et al. Kiloparsec-scale Properties of Emission-line Galaxies. ApJ 797, 108 (2014).

5. Schaefer, B. E. Comprehensive Photometric Histories of All Known Galactic Recurrent Novae. ApJS 187, 275-373 (2010).

6. Darnley, M. J. et al. A remarkable recurrent nova in M 31: The optical observations. A\&A 563, L9 (2014).

7. Yaron, O., Prialnik, D., Shara, M. M. \& Kovetz, A. An Extended Grid of Nova Models. II. The Parameter Space of Nova Outbursts. ApJ 623, 398-410 (2005).

8. Kato, M., Saio, H. \& Hachisu, I. Multi-wavelength Light Curve Model of the One-year Recurrence Period Nova M31N 2008-12A. ApJ 808, 52 (2015).

9. Henze, M. et al. A remarkable recurrent nova in M 31: The predicted 2014 outburst in X-rays with Swift. A\&A 580, A46 (2015).

10. Wambsganss, J. Quasar Microlensing. In Brainerd, T. G. \& Kochanek, C. S. (eds.) Gravitational Lensing: Recent Progress and Future Go, vol. 237 of Astronomical Society of the Pacific Conference Series, 185 (2001).

11. Kochanek, C. S. Quantitative Interpretation of Quasar Microlensing Light Curves. ApJ 605, 58-77 (2004).

12. Paczynski, B. Gravitational microlensing by the galactic halo. ApJ 304, 1-5 (1986).

13. Alcock, C. et al. Possible gravitational microlensing of a star in the Large Magellanic Cloud. Nature 365, 621-623 (1993).

14. Aubourg, E. et al. Evidence for gravitational microlensing by dark objects in the Galactic halo. Nature 365, 623-625 (1993).

15. Udalski, A. et al. The optical gravitational lensing experiment. Discovery of the first candidate microlensing event in the direction of the Galactic Bulge. ACTAA 43, 289-294 (1993). 
16. Chang, K. \& Refsdal, S. Flux variations of QSO 0957+561 A, B and image splitting by stars near the light path. Nature 282, 561-564 (1979).

17. Chang, K. \& Refsdal, S. Star disturbances in gravitational lens galaxies. A\&A 132, 168-178 (1984).

18. Miralda-Escudé, J. The magnification of stars crossing a caustic. I - Lenses with smooth potentials. ApJ 379, 94-98 (1991).

19. Planck Collaboration et al. Planck 2015 results. XIII. Cosmological parameters. A\&A 594, A13 (2016). 